\title{
Spatial and temporal shaping of diffuse thermal wave fields using high-power lasers
}

\author{
by M. Ziegler*, S. Ahmadi*, P. Hirsch, C. Hassenstein*, E. Thiel* , N. W. Pech-May* \\ *Bundesanstalt für Materialforschung und -prüfung (BAM), 12200 Berlin, Germany, mathias.ziegler@bam.de
}

\begin{abstract}
Although thermography is suitable for a wide range of inhomogeneities and materials, the fundamental limitation is the diffuse nature of thermal waves and the need to measure their effect radiometrically at the sample surface. The crucial difference between diffuse thermal waves and propagating waves is the rapid degradation of spatial resolution with increasing depth. A promising approach to improve the spatial resolution and thus detection sensitivity and reconstruction quality lies in shaping of these diffuse thermal wave fields. We present the latest results of this technology obtained with lasers, i.e. spatially and temporally structureable heating sources and modern numerical methods.
\end{abstract}

\section{Introduction}

Newton's well-known cooling law obeys an exponentially decreasing temperature. This is the consequence of the underlying differential equation, which is closely related to the diffusion equation. In this context it is therefore also said that the heat "diffuses". If we do not heat a material constantly or pulsed but modulated, the heat conduction follows this modulation. For the differential equation we have to formulate the boundary values oscillating in time, for example sinusoidal. The solution is a strongly damped thermal wave. It is called this way because this special solution is very similar to the solutions of the wave equation.

Anders Jonas Ångström discovered this exciting property experimentally in 1861 and explained it theoretically [1] $\mathrm{He}$ alternately used steam and ice water to heat a long metal rod and measured the alternating temperature fluctuation at different distances on the rod with thermometers. He was able to determine the thermal diffusivity $\alpha$ from the relationship between distance and delay with which the thermal wave passed the thermometers.

The concept of wave is of such fundamental importance for the way of thinking in applied and pure physics that the transfer of wave phenomena to diffusion processes has led to the new class of diffusion waves [2, 3]. In contrast to the wave equation, the diffusion equation has only a time derivative of the first order instead of the second order. As a consequence there are also important differences between the thermal waves and the propagating plane waves as they are known from mechanics or electromagnetism. For example, the spatial attenuation is so high that the thermal wave can practically no longer be detected after only one wavelength [4]. An analogous relationship can be found in the skin effect, where electromagnetic waves penetrate only minimally into a good conductor. In addition, thermal waves are strongly dispersive. Their phase velocity is directly dependent on the modulation frequency of the heat source. Therefore, it is not possible to produce propagating wave packets, since each partial wave propagates at its own phase velocity.

\section{Non-destructive testing using thermal waves}

Non-destructive material testing makes use of both effects, material-dependent damping and thickness dependent phase shift. An impressive example is the quality control of carbon fibre composites (CFRP) and coatings. Here the surface is periodically illuminated with a light source [5]. If a planar light source such as a halogen lamp is used for illumination and a thermographic camera for temperature measurement, this is referred to as active thermography. If, on the other hand, a focused laser beam plus a photodetector is used to scan the sample, this is referred to as photothermal layer thickness measurement. Both types are modern variants of the Ångström experiment: the warm-cold water change is exchanged for a modulated light source and the thermometers for a thermography camera or a photodetector.

If we now want to determine the quality of the layer system, we have to consider the thermal wave, which decays to $1 /$ e after the thermal diffusion length. If, however, it encounters another material within this diffusion length, for example air, because of delamination, heat accumulates at the interface due to its lower thermal conductivity, density and specific heat capacity. This heat accumulation can be understood as a reflection of the thermal wave. The wave trains running back and forth overlap and interference occurs. The temporal temperature curve at the surface exactly above the material defect, i.e. the phase information, changes characteristically. It allows a precise determination of the thickness of the layer passing through and the extent of the defect [6]. This principle can be immediately extended to a multitude of materials and defects, such as gas inclusions in metals (blowholes) or cracks in concrete.

Thus, information about the interior of solids can be obtained by temporal shaping of thermal waves. The depth into which active thermography can penetrate depends on the material, but usually remains in the range of tenths of a millimetre to a few millimetres, in extreme cases to a few centimetres. In principle, in order for a defect to be detectable, its expansion parallel to the sample surface must be at least as large as the depth at which it lies. 


\section{Spatial and temporal shaping of diffuse thermal wave fields using high-power lasers}

A completely new approach that we are currently working on is the shaping of thermal waves as thermal wave fields not only on the time axis, but in any spatiotemporal structure. This will enable us to locate material defects even more precisely. The most important theoretical prerequisite for this is the linearity of the heat conduction equation in space and time. Mathematically it is a linear partial differential equation of second order. Under this assumption, the superposition principle applies, which states that a linear combination of solutions of the linear equation is also a solution.

In practical terms, this means that a structured thermal wave field can be generated from the thermal waves of individual heat sources which are themselves arranged in a certain structure. As early as 1822, Jean Baptiste Joseph Fourier solved the heat conduction equation he had discovered by making use of its linearity [7]. The linear Fourier transformation developed by him for this purpose essentially states that any spatiotemporal pattern can be represented as a superposition of harmonic, i.e. sinusoidal and cosinusoidal, waves of different frequencies.

As we know it from optics or mechanics, thermal waves can be interfered with each other constructively or destructively. Although any heat source can in principle be used in active thermography, optical sources are particularly suitable because they enable a completely non-contact test procedure: Besides the infrared camera we only need a light source. For the technical realization, controllable individual sources are required, which must have certain properties. The most important property of the partial waves is their coherence. This means that there must be a fixed phase relationship between them. In practical terms, this usually does not mean a major restriction for thermal waves, since the frequency range being considered can be managed with standard measurement technology: it ranges from the millihertz to the kilohertz range, which corresponds to thermal wavelengths from the centimeter to the micrometer range. Only the light source itself must be quickly switchable and modulatable.

In addition, a linear dependence of its output power on the control signal is advantageous, because harmonic heating via a harmonic control signal is then possible. Furthermore, a light source with controllable irradiation distribution should be used and have an emission wavelength that differs from the sensitivity range of the thermographic camera. This last point is particularly important when metals with high reflectance have to be examined. Otherwise, there may be a confusion between the thermal radiation emitted by the sample, i.e. the real measurement signal, and the excitation radiation reflected by the sample.

All properties listed here can be realized with laser sources. In particular, diode lasers are the optical sources of choice because they are inexpensive, practically maintenance-free and easy to operate. An obvious approach is to project a high-power diode laser onto the sample via a micro-mirror array (Digital Micromirror Device, DMD), which can be found in many high-end video projectors. Using the DMD, we can illuminate any location with pixel precision. This enables us to generate individual thermal wave fields and to superimpose thermal waves in a targeted and coherent manner. We have shown experimentally that this approach works in principle [8, 9]. Unfortunately, it also has some weak points. The coupling of the laser beam is very difficult, and the DMD is not designed for the achievable power of high-power diode lasers .

If we use a laser array instead, we can generate several orders of magnitude more power. This approach currently still has the weak point that there are no powerful 2D arrays with more than $100 \mathrm{~W}$ power so far. Only recently have 1D arrays become commercially available. These consist of several line emitters arranged side by side, which can only be controlled in their entire line, i.e. one-dimensionally. With these vertical-cavity surface-emitting laser (VCSEL) arrays we have so far been able to realize some basic experiments on thermal waveforming.

In one approach $[10,11]$ we have let two wave fields oscillate antiphasically and destructively interfere with each other. The resulting high sensitivity was used to detect vertical crack-like defects below the surface, which can only sometimes be found with classical active thermography. In another approach [12], closely adjacent defects could be separated by multiple measurements with varying illumination structures. We present the latest results of this technology obtained with lasers, i.e. spatially and temporally structureable heating sources and modern numerical methods.

Gefördert durch die Deutsche Forschungsgemeinschaft (DFG) - 400857558, funded by the Deutsche Forschungsgemeinschaft (DFG, German Research Foundation) - 400857558.

\section{REFERENCES}

[1] A. J. Ångström, Ann. Phys. Lpz. 1861, 114, 513.

[2] A. Mandelis, Diffusion-Wave Fields, Springer Verlag, New York 2001.

[3] A. Mandelis, Physics Today 2000, 53(8), 29.

[4] O. Breitenstein et al., Lock-in Thermography, Springer Verlag, Berlin 2010.

[5] C. A. Bennett et al., Appl. Opt. 1982, 21(1), 49.

[6] D. P. Almond et al., Photothermal Science and Techniques, Chapman \& Hall, London 1996.

[7] J. B. J. Fourier, The Analytical Theory of Heat, The University Press, London 1878.

[8] E. Thiel et al., J. Vis. Exp. 2017, 123, e55733.

[9] E. Thiel et al., Appl. Phys. Lett. 2016, 109(12), 123504.

[10] M. Ziegler, E. Thiel, T. Studemund, Materials Testing: Vol. 60, No. 7-8, pp. 749-757, 2018.

[11] E. Thiel, M. Ziegler, T. Studemund, Int J Thermophys 40, 17, 2019.

[12] P. Burgholzer, T. Berer, M. Ziegler, E. Thiel, S. Ahmadi, J. Gruber, G. Mayr \& G. Hendorfer, Quantitative InfraRed Thermography Journal, DOI: 10.1080/17686733.2019.1655247, 2019. 